\title{
Gold Bonding Wire for Semiconductor Applications
}

\author{
Susumu Tomiyama and Yasuo Fukui
}

Tanaka Denshi Kogyo Co. Limited., Mitaka-shi, Tokyo, Japan

Because of the ease with which gold wire can be bonded by modern techniques, it is used extensively for making connections to or between the active components in semiconductor devices such as transistors and integrated circuits. This article reviews some of the important properties of gold bonding wire and discusses trends in its use in industry.

As a result of its widespread use in bonding applications in semiconductor devices, many products such as computers, numerical control machines, medical instruments, automobiles, television sets and electronic cameras contain gold wire. Despite intensive attempts to find substitute materials, the choice of gold for this application has not so far been seriously challenged. The special merits of gold wire include its resistance to tarnish and corrosion, its high electrical conductivity and, especially, the relative ease with which it can be bonded into position by thermocompression and ultrasonic bonding techniques.

Figure 1 shows the regular increase in the amounts of gold wire consumed annually in Japan over the past five years. Because of the increase in the price of gold in late 1979 and early 1980, however, intensive efforts have been made to reduce the diameter of the gold wire used, while maintaining or improving the efficiency with which it can be bonded into position in electronic devices.
As a result, changes are already evident in the amounts of gold wire in the diameter ranges which are consumed in Japan. Data in this connection for the first quarters of 1980 and 1981 are presented in Figures 2 (a) and 2 (b) respectively. It can be seen from these how, over a period of one year, the consumption of $25 \mu \mathrm{m}$ diameter gold wire, conventionally used in small-signal transistor integrated circuits, decreased and that of 20 and $23 \mu \mathrm{m}$ diameter wire increased.

A further development has been stimulated by the availability of very high speed automated bonding machines, in which bonding of wire to form a loop is completed in less than $0.2 \mathrm{~s}$. Since, in these machines a spool of $100 \mathrm{~m}$ of gold wire (which weighs approximately $1 \mathrm{~g}$ in the case. of $25 \mu \mathrm{m}$ diameter wire) was finished in only half a day, a greater length of wire per spool was called for. Moreover, consistency in the quality of the wire, not only on each spool, but also from spool to spool, became of increased importance if the high efficiency of the new machines was to be fully exploited.

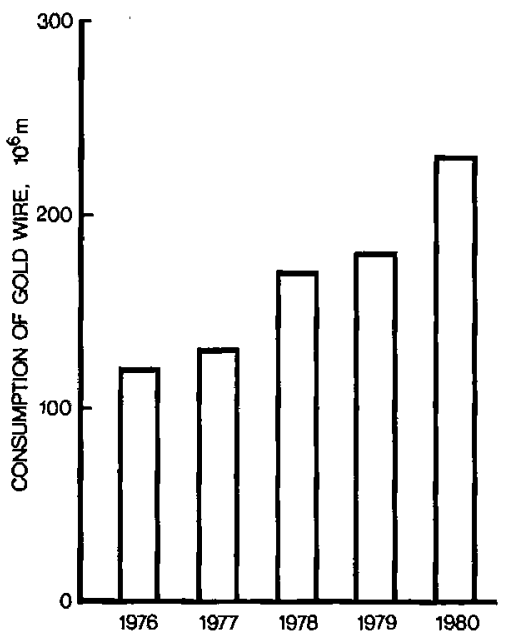

Fig. 1 Increase in gold wire consumption in Japan from 1976 to 1980
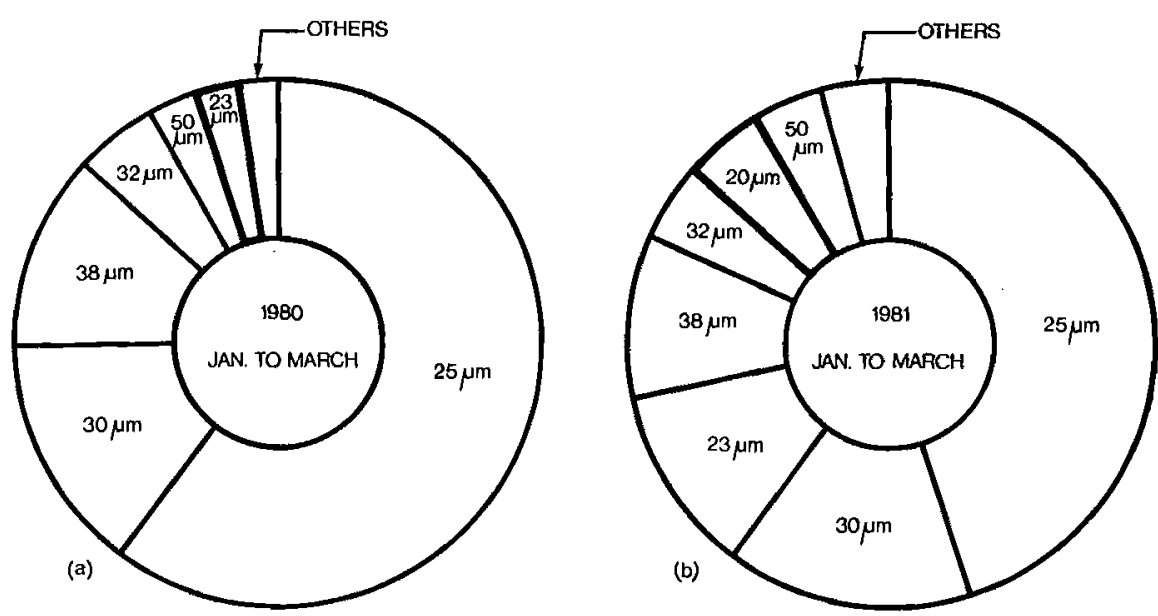

Fig, 2 Consumption of gold wire of diffetent diameter in Japan in 1980 and 1981 over the January-March period 


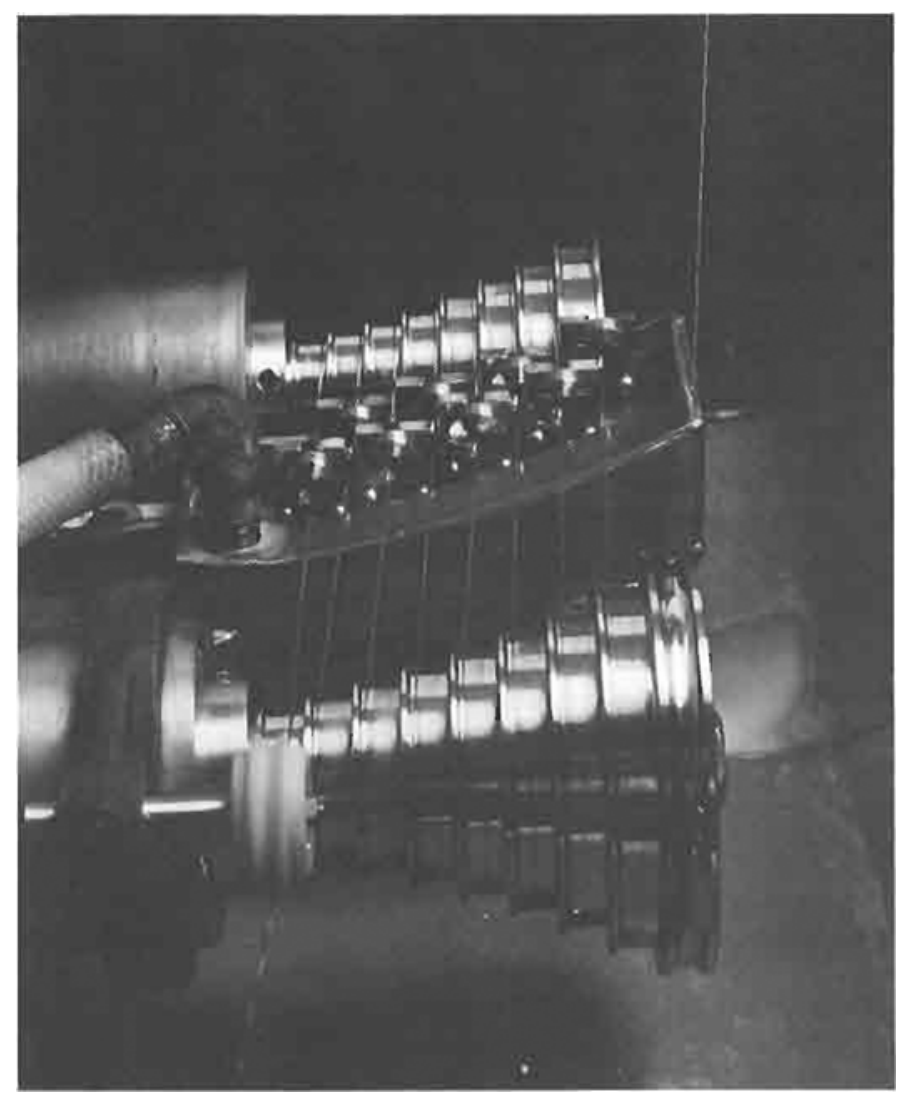

Fig. 3 A typical modern wire-drawing apparatus

This paper describes, against the above background, the effects of a range of dopant elements upon the characteristics of gold wire of 99.99 to 99.999 per cent purity. These have been significant in our efforts to develop fine gold wire of improved stability.
The effects of minute additions of other elements to gold have been described by others, in particular the effects of doping it with beryllium ( 1 to 4 ), copper (3), carbon (5) and yttrium $(4,6,7)$.

\section{Production}

When wire is drawn, energy is stored in the metal as a result of the formation of dislocations and lattice defects. This strain energy is released when the metal recrystallizes and its magnitude, as determined by the degree to which the metal has been worked, affects the temperature at which recrystallization occurs in pure gold wire in storage or during use. Because recrystallization is accompanied by grain growth and loss of mechanical strength, efforts to produce improved gold wire have been focussed upon a search for elements which when incorporated in gold ir trace quantities not only exercise a grain refining effect, but also raise the recrystallization temperature.

The gold content of wire for semiconductor devices is usually more than 99.99 per cent (8). The starting material for its manufacture is commercially available gold of this purity, which is first refined in order to eliminate undesired or unnecessary impurities. The product of that operation is 99.999 per cent pure and dopants are added to it in strictly controlled amounts in order to obtain material which is suitable for wire production.

Table I illustrates how the recrystallization temperature $\left(150^{\circ} \mathrm{C}\right)$ of gold wire of more than 99.999 per cent purity is affected by the incorporation in it under standardized conditions of a variety of dopants in concentrations of less than $50 \mathrm{ppm}$. It will be noted that dopants such as aluminium, beryllium, calcium, lead, thallium and tin raise the recrystallization temperature above $300^{\circ} \mathrm{C}$, while silver, platinum and palladium do not affect it at all. The effects of certain other elements are intermediate in magnitude.

The actual manufacture of bonding wire consists of melting, casting and working gold of the appropriate composition through calibrated rolls $(3,9)$. Thereafter, a coarse strand, approximately $1 \mathrm{~mm}$ in diameter, is produced in a single drawing operation and this is continuously cold-drawn through a succession of lubricated dies until the desired diameter is reached. The drawn wire is then heat-treated to adjust its mechanical strength to the level desired, and the finished product is then rewound on spools. The process of, and equipment used, in drawing are illustrated in Figure 3. 

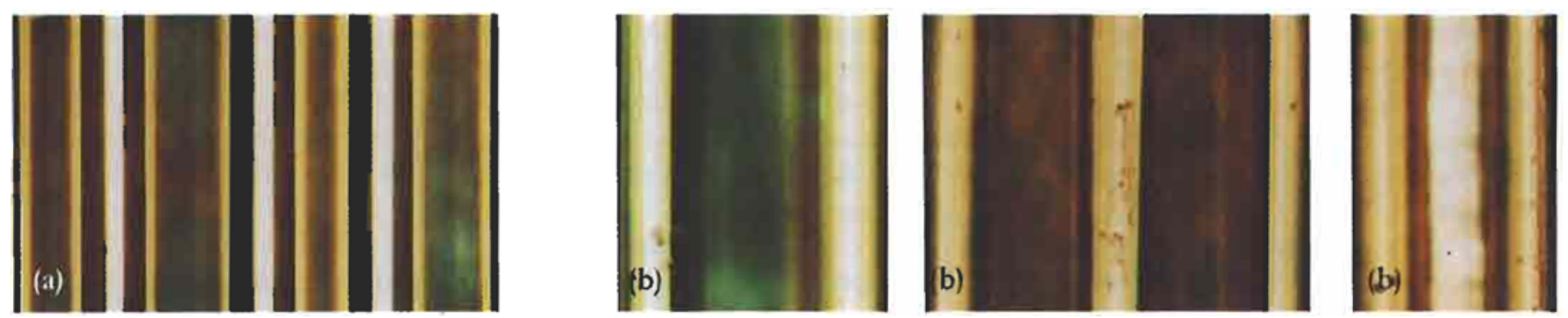

Fig. 4 Wires produced in atmospheres of controlled, (a), and uncontrolled, (b), cleanliness

Fig. 5 Schematic representation of a thermocompression bonder

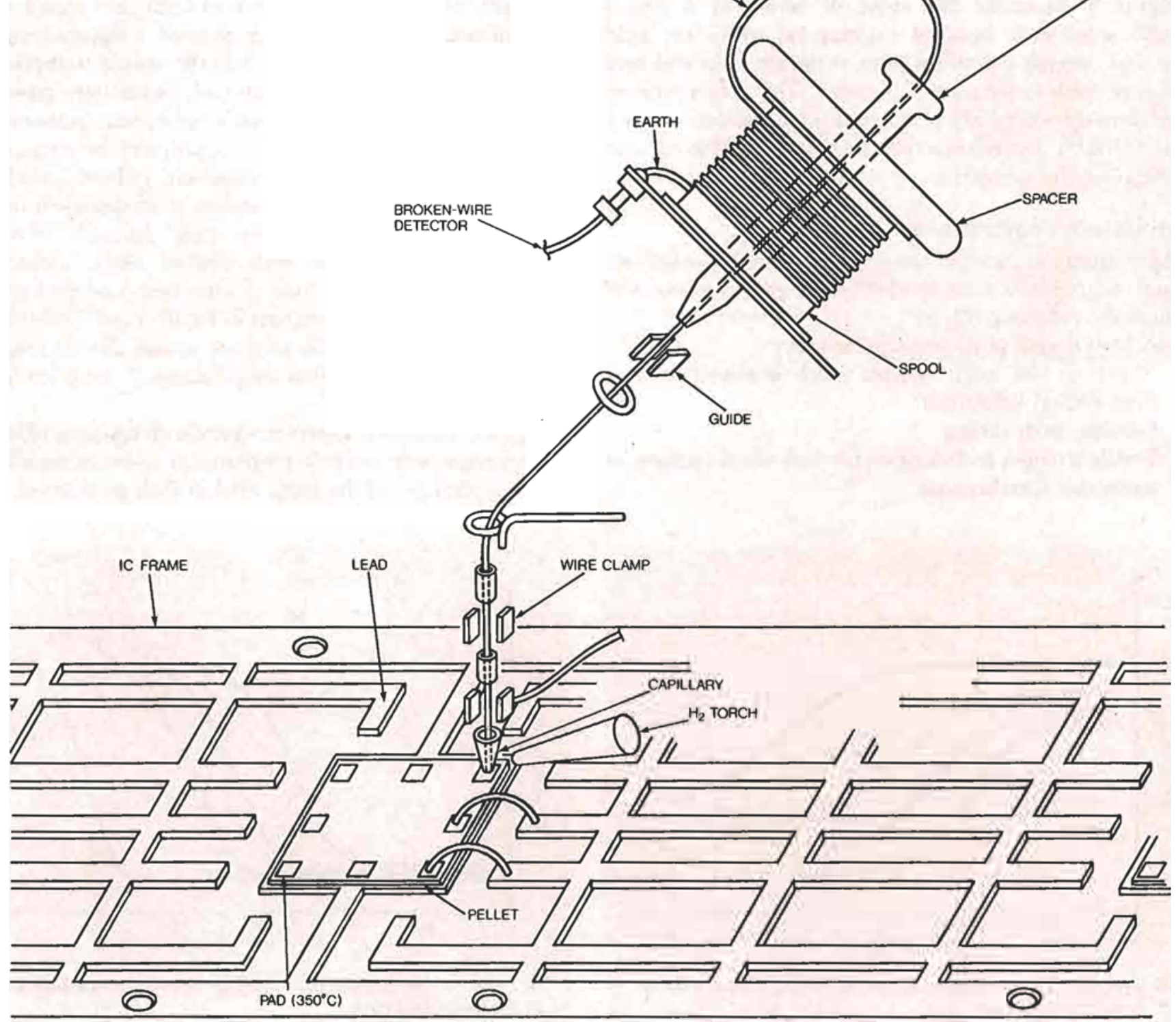


As gold wire for bonding must have a smooth, uncontaminated surface, it is essential that all production operations be performed in a clean environment. Thus, Figure 4(a) illustrates the appearance of gold wires produced in a room which was maintained in a clean condition, while Figure 4(b) illustrates that of wires produced in a toom the cleanliness of which was not controlled.

Since a variety of machines is available for high speed bonding of gold wires, and these machines are constantly being modified and improved, it is necessary from time to time to modify production methods so as to produce wires for particular machines or to develop production methods and specifications for wires for new types of machines.

Figure 5 illustrates the mode of action of a typical thermocompression bonding machine on which the gold wire, fed through a capillary tube, is melted at its end by a hydrogen torch to form a ball of metal. This is first pressure bonded to the wiring pad of the semiconductor chip on a hot plate at $350^{\circ} \mathrm{C}$, looped smoothly and then bonded to the lead to complete the connection.

\section{Performance Requirements}

Apart from the purity of the gold used in its manufacture, requirements which must be satisfied by gold bonding wire include the following (8):

(1) A high degree of dimensional accuracy

(2) A smooth and bright surface which is completely free from foreign substances

(3) Freedom from curling

(4) Tensile strength and elongation values which conform to acceptable specifications

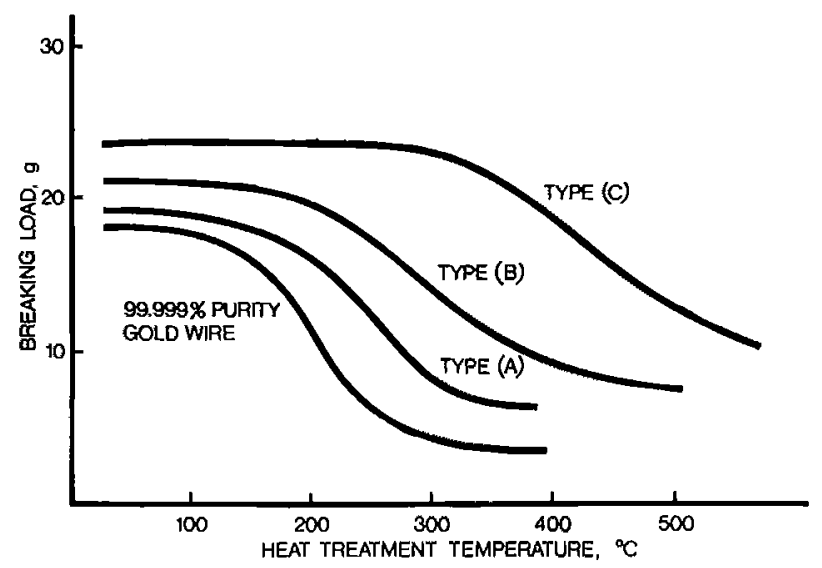

Fig. 6 Relation between breaking load and heat treatment temperature for $25 \mu \mathrm{m}$ diameter gold wire
(5) Production of bonds which consistently conform in strength to acceptable standards

(6) Formation of loops of satisfactory and consistent shape.

Of the six items quoted above, the last three are highly dependent on doping, and have been studied from this aspect.

\section{Tensile Properties}

In respect of tensile strength and elongation, the most critical aspect is the extent to which they are affected by exposure of the wire to heat. This is determined essentially by the degree to which the recrystallization temperature of the gold has been increased by the dopants incorporated in it. Some observations in this connection, made on wires of $25 \mu \mathrm{m}$ diameter, which had been fabricated from gold doped with traces of various other metals, are recorded in Figures 6 and 7 .

From Figure 6 it is evident that the tensile strengths of wires made from gold doped with lead, aluminium, calcium, thallium, tin or beryllium are unaffected by heat treatment at temperatures up to $300^{\circ} \mathrm{C}$. The same property for wires made from gold doped with iron, germanium, gallium, cobalt or copper is unaffected by heat treatment at temperatures up to $200^{\circ} \mathrm{C}$. On the other hand, the tensile strengths of wires made from gold doped with silicon, silver, nickel or palladium are similar to those of wires from undoped gold.

It will also be noted from data in Figure 6 and Table I that the tensile strengths of the wires are almost directly proportional to the recrystallization temperatures of the golds from which they are made.

Figure 7 illustrates, however, that the elongations of wires are approximately inversely proportional to the recrystallization temperatures of the golds used in their production.

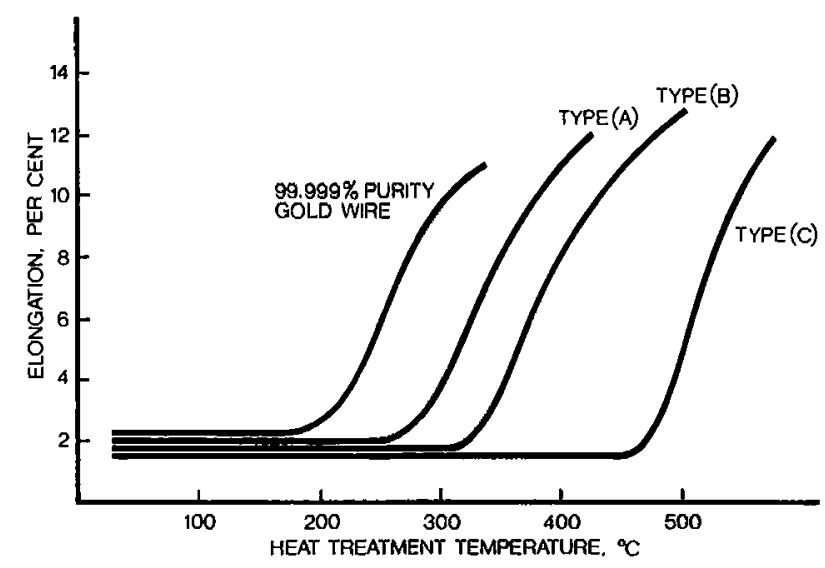

Fig. 7 Relation between elongation and heat treatment temperature for $25 \mu \mathrm{m}$ diameter gold wire 


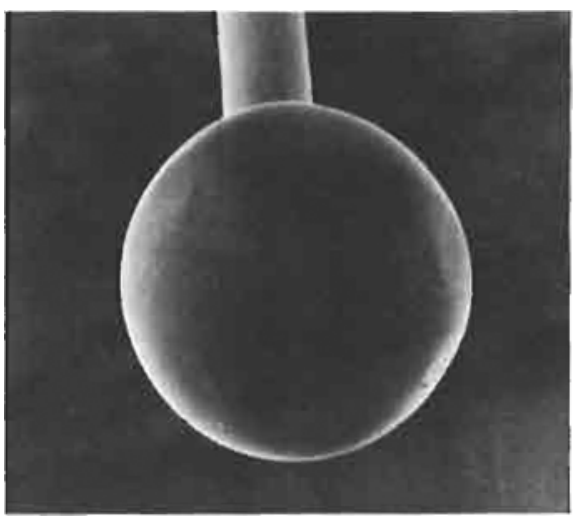

Fig. 8 A typical well-shaped ball generated from gold wire of 99.999 per cent purity

Fig. 9 Various defective ball shapes (Types A to $\mathrm{E}$ ) formed from gold wire of 99.99 per cent purity containing various dopants
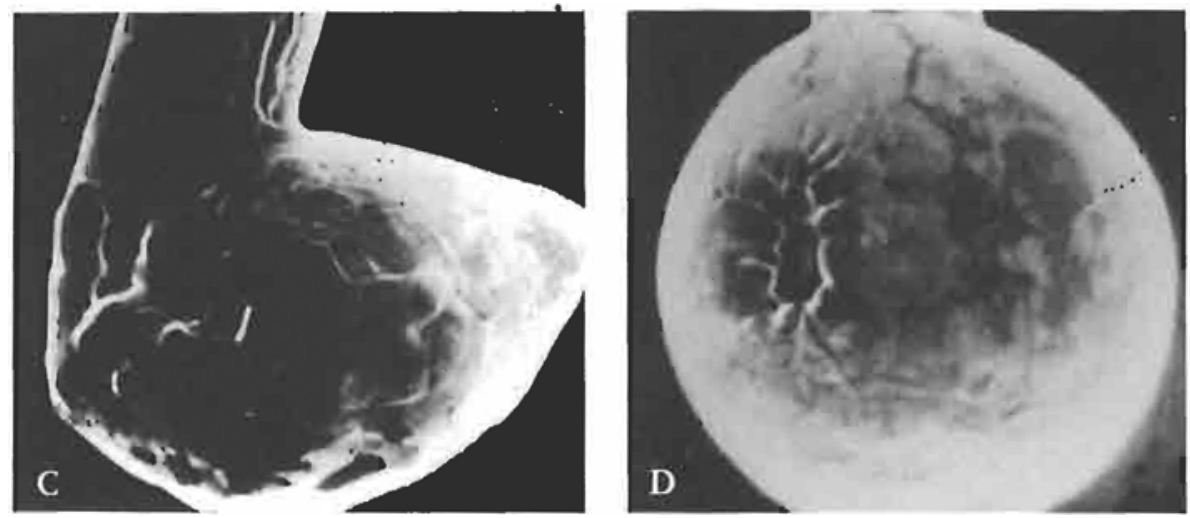

Some undesirable ball shapes are illustrated in Figure 9 and the following comments may be made on these:

(1) In Type A, the tip of the gold wire does not form a ball of metal when heated with the hydrogen torch of a bonding machine. Dopants such

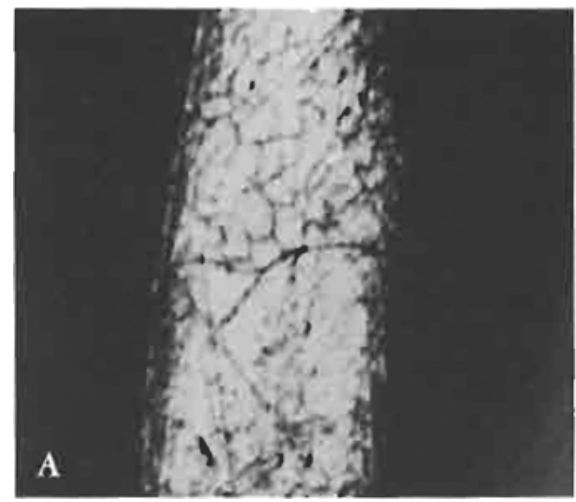

as aluminium and silicon give rise to this effect

(2) In Type B, which is similar, dopants such as indium and tin may be the cause

(3) In Type C, in which the ball which is formed is distorted in shape, palladium or platinum may be present as dopants

(4) In Type D, in which furrows are formed on the ball surface, the cause may be the use of dopants such as lead or thallium

(5) In Type E, in which oxide films are formed on the ball surface, dopants such as copper, iron, cobalt, magnesium or gallium may be the cause.
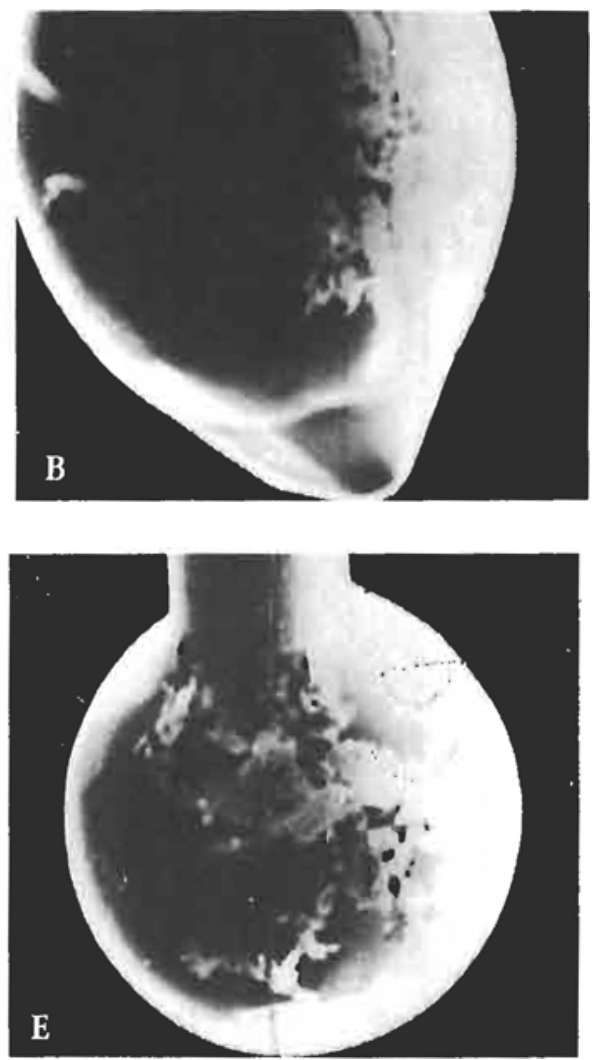


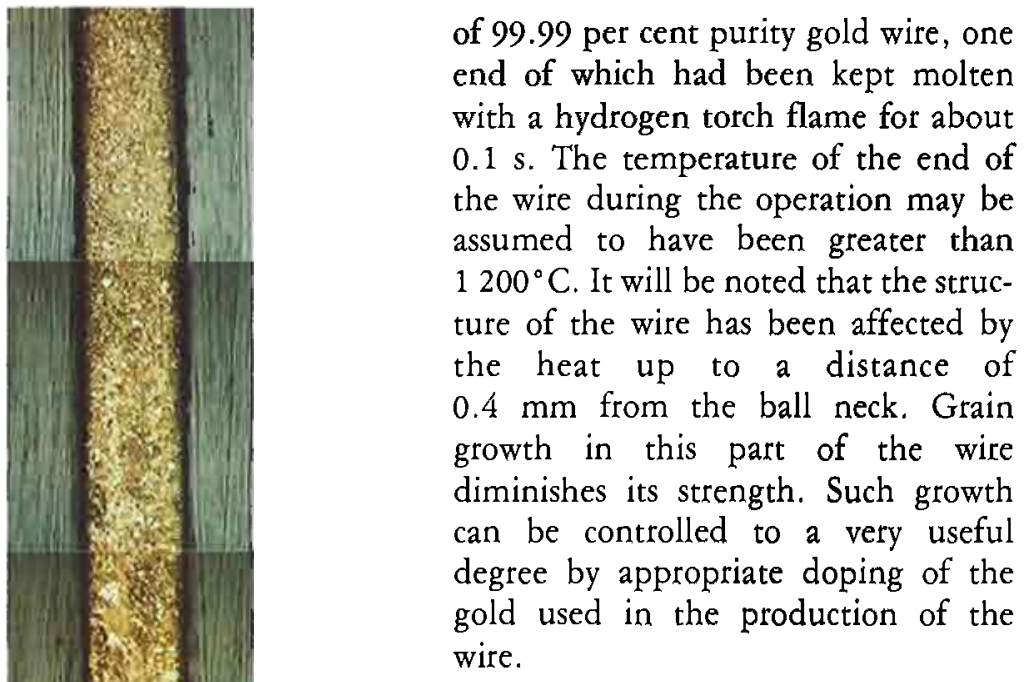

\section{Loop Shape}

The shape of the loop formed during bonding depends essentially upon the setting of the bonding machine used, the shape of capillary through which the wire is fed and the properties of the bonding wire itself. It is usual to optimize the loop shape by altering or adjusting the travel of the capillary point between the two bonds. By changing both the length and the hieight of this travel between the points to be connected the loop shape can be improved. The problems associated with inappropriate loop shape are neck failure, tab-shorting and tailing as shown in Figure 11.

The effects of using wires made from gold doped in various ways upon the incidence of these problems has been studied. It was found that impurities in the gold can be well classified into three groups in this respect. Thus, lead, aluminium, gallium, thallium and bismuth are often a cause of neck breakage, whereas lead, tin and indium are closely associated with the occurrence of tab-shorting, which is critical for the finished package. Platinum, palladium, silver and silicon tend to produce tailing. The consequence of this latter problem is that, depending on the tailing size, the formation of the next ball may be affected with the result that it forms an unstable bond.

\section{Bond Strength}

Bond strength may be measured by the technique illustrated in Figure 12. A tension gauge of a type commonly available is used. In testing the strengths of the bonds of a wire loop, the latter is hooked at its mid-point and pulled at a constant velocity of $0.5 \mathrm{~cm} / \mathrm{s}$.

Fig. 11 Defects occurring in gold wire due to bad loop shape

Fig. 10 Grain growth in the portion of a gold bonding wire heated during ball formation

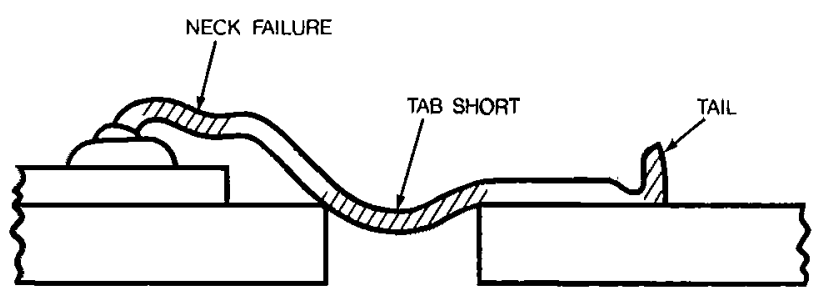


Fig. 12 Method used for measuring gold wire bond strength

Using this method, a number of effects were observed. Thus, in cases where the surface of the gold oxidizes during torch melting, the strengths of the bonds are low and the wire tends to peel off at its bonding interface with the pad or lead. In other instances, failure occurs at the ball neck as a result of grain growth and segregation of impurities at grain boundaries at this point (10). Figure 13 illustrates a failure of the latter type.

\section{Discussion}

Commercially available gold wires normally contain intentionally added trace elements together with traces of other elements present as 'adventitious' impurities. Wires from various suppliers which appear to be of very similar composition may therefore perform differently in practice. In the present state of the art, however, the mechanical properties of gold bonding wires, and their tensile strengths in particular, are amenable to control by correct selection of dopant species and concentrations.

As a result of appropriate doping, the two wires marked A and $B$ which are shown in Figure 14 have significantly improved tensile strengths compared to pure gold wire. These two types of wires are of the quality most commonly used in assembly operations in Japan. The wire diameter normally ranges from 10 to $50 \mu \mathrm{m}$. The significant advantage afforded by doping is that the same tensile strengths can be obtained at a smaller diameter which facilitates bonding of wires on smaller pads. Cost savings are also achieved in material usage.

\section{Concluding Remarks}

The increase in the gold price which occurred in late 1979 and early 1980 has had an impact on the industries which use semiconductors. Thus, copper materials, gold-silver alloys and gold clad materials have been evaluated as substitutes for gold wire in some applications. However, these have not yet been successful in replacing gold and the effort to economize is directed towards decreasing gold consumption by using finer wires and smaller loops. For example, most gold wire used for small transistor integrated circuits is now less than

Fig. 14 Improved tensile properties of appropriately doped gold wires
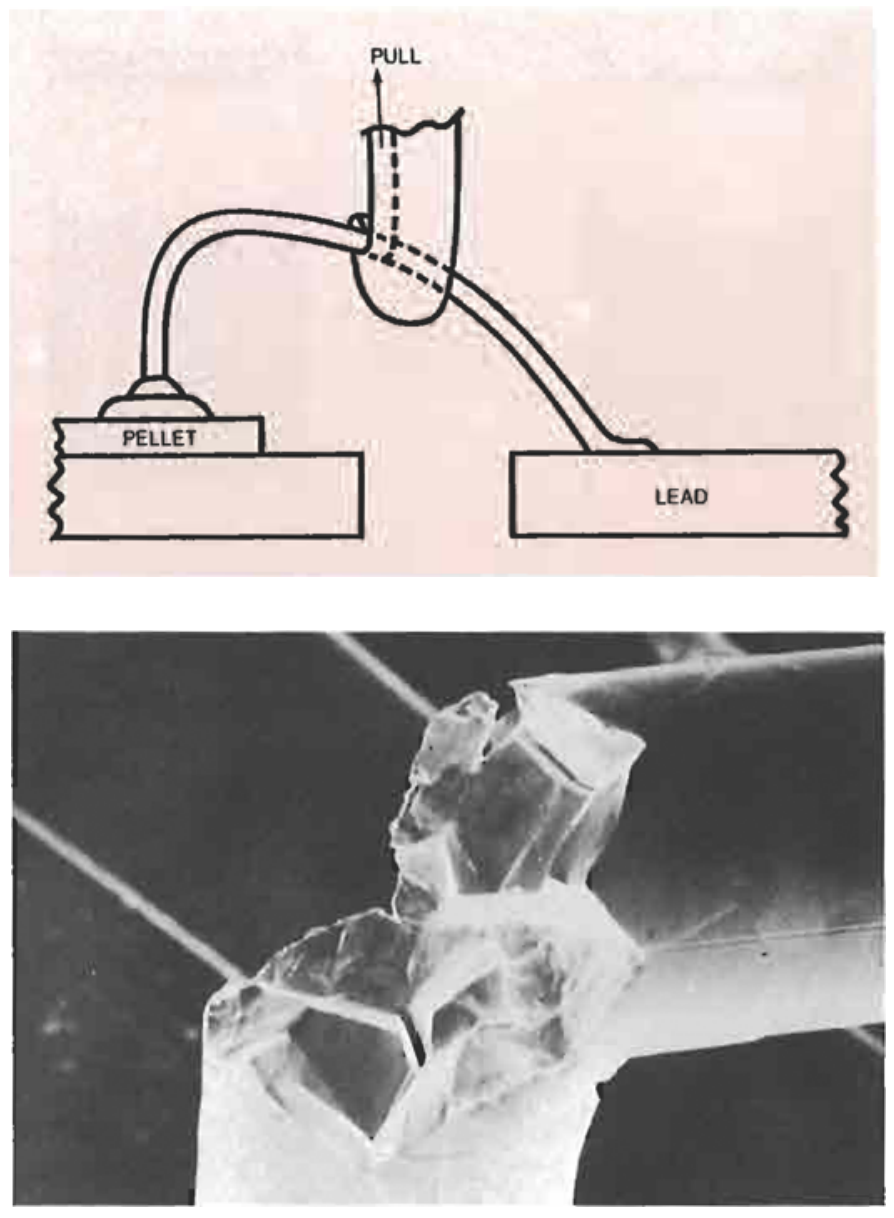

Fig. 13 Photonicrograph of grain boundary failure at the ball neck

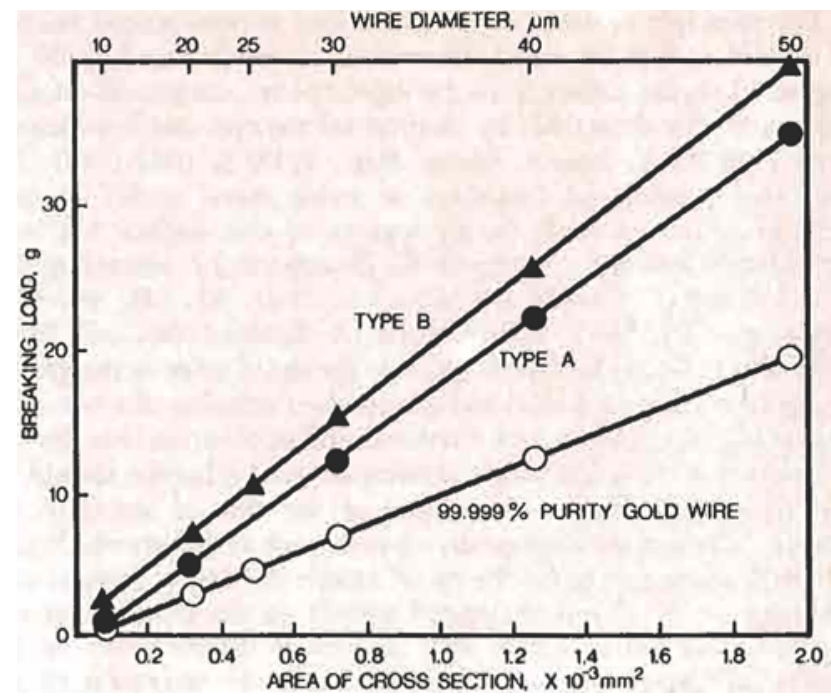



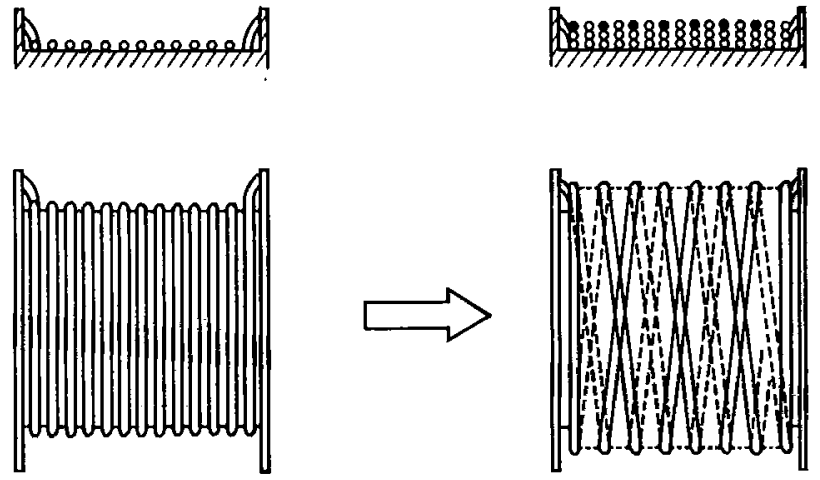

$20 \mu \mathrm{m}$ in diameter. Furthermore, the need for increasing the length of gold wire on a single spool has become pressing with the introduction of high speed automated bonding machines. This will reduce the frequency of changing spools and raise manufacturing efficiency. It is possible to increase this length by replacing the standard $5 \mathrm{~cm}$ spools by spools of larger diameter or width. However, the adoption of new processes and materials implies further investment. Consequently, as Figure 15 shows, multilayer winding causing no damage
Fig. 15 Conventional single-layer and modern multiple-layer winding techniques.

to the wire has been devised. Single lengths of 500 and 1000 $\mathrm{m}$ are already available commercially.

Thus, in the immediate future, the main thrust of development will remain directed towards the promotion of finer gold wires and increased length per spool which improves their cost/performance ratio.

\section{References}

1 B. Brenner, U.S. Patent 3,272,625 (1966)

2 T.H. Ramsey, Solid State Technol., 1973, 16, (10), 43-47

3 B.L. Gehman, Solid State Technol., 1980, 23, (3), 84-91

4 W.S. Rapson and T. Groenewald, 'Gold Usage', Academic Press, London, 1978

5 M. Bonkohara, U.S. Patent 4,080,485 (1978)

6 C. Raub, M. Thiede and H. Thiede, Ger. Patent $1608161 \cdot(1971)$

7 H.H. Daut, K. Edelmann, K. Schwarz and J. Bolik, Ger. Dem. Rep. Patent 126550

8 ASTM, 'Standard Specification for Gold Wire for Semiconductor LeadBonding', F72-74

9 H.P. Thiede and A. Bischoff, 'Proc. Int. Microelectron. Symp.', 1979 $77-83$

10 H.K. James, IEEE, Trans. Components Hybrids Manuf. Technol., 1980 CHMT-3, (3), 370-374

\section{Gold Aids Crystal Surface Studies}

The principle of detecting and revealing microstructural features of a solid surface by metal decoration, in which metal atoms are deposited on the surface from the vapour phase, coalesce and form a pattern readily detectable by electron microscopy, has been known since 1958 (G.A. Bassett, Pbilos. Mag, , 1958, 3, 1042-1045). The idea that preferential formation of stable metal nuclei on solid surfaces occurs on high energy regions of that surface has been extended in a recent study by D.W. Thompson, J.J. Macmillan and D.A. Wyatt (J. Colloid Interface Sci., 1981, 82, (2), 362-372). Earlier work by V.M.E. Robinson and J.L. Robins (Thin Solid Films, $1974,20,155-175)$ had indicated that the initial stage in the growth of a gold particle on a solid surface was the formation of a one-atom critical nucleus. This has the mathematical implication that the rate of formation of small stable clusters of gold adatoms should be directly proportional to the square of the flux of atoms to the surface. When nucleation occurs on sites, such as defects which have a high binding energy for the metal atoms, the rate of formation of adatoms can be shown to depend linearly on the adatom flux and the concentration of defect sites, as well as exponentially on the energy of defect sites in the surface and the reciprocal of the substrate temperature.
Thompson and his co-workers studied the coalescence of vapourdeposited gold atoms on different layer-lattice silicates in order to assess the uniformity of the crystal surface structure of these minerals. The feasibility of using this technique to obtain a quantitative measure of the defect nature on silicate surfaces, on the basis of the above considerations, was also studied.

The most successful results were obtained using muscovite and montmorillonite on which the distribution of gold particles was found to be reasonably uniform. Plots of gold particle population density against evaporation time showed that, for given substrate temperatures, the rate of particle formation for the two mineral surfaces, assuming that substitutional atoms in these surfaces constitute point defects at which preferential nucleation of gold particles occurs, is consistent with the relative extents of isomorphous substitution in the silicate sheets.

It would appear, therefore, that the rate of nucleation of gold particles on certain surfaces is determined by the concentration in them of substitutional atoms. This interesting and unusual phenomenon, and the principles behind it, are promising enough to merit continued study. 